\title{
Colesteatoma em cães: revisão de literatura
}

\section{Cholesteatoma in dogs: literature review}

\section{Resumo}

Colesteatoma é uma doença otológica caracterizada por um cisto epidermoide aural benigno. As manifestações clínicas apresentadas por essa doença podem ser: otalgia, dor ou inabilidade ao abrir a boca, head tilt, paralisia facial, ataxia e dor à palpação da articulação temporomandibular. O diagnóstico se baseia em exames como tomografia, ressonância magnética, vídeo-otoscopia, sendo fundamental realizar o histopatológico para confirmação do diagnóstico. A cirurgia é o tratamento mais eficaz, podendo não ser curativo. A cirurgia consiste em remover os restos de queratina e epitélio escamoso da orelha média, e na Medicina Veterinária tem alto índice de recidiva.

\section{Abstract}

Cholesteatoma is an otologic disease, characterized as a benign aural epidermoid cyst. Clinical manifestations of this disease can be: otalgia, pain or inability to open the mouth, head tilt, facial paralysis, ataxia, and pain under temporomandibular joint palpation. Diagnosis is based on exams such as tomography, magnetic resonance imaging, and video-otoscopy; and histopathological examination is essential to confirm the diagnosis. Surgery is the most effective treatment, albeit it may not be curative, consisting of removing the remains of keratin and squamous epithelium of the middle ear. In Veterinary Medicine, this has a high rate of recurrence. 
Tamires De Vecchi Trani ${ }^{1}$

Natália Guerreiro Lyra²

Bruno Pietroluongo ${ }^{3}$
Rua Octávio Vannini, 273,

Parque dos Príncipes, São Paulo/SP, Brasil

CEP: 05396-455

凶tami_vecchi@hotmail.com

\section{Palavras-chave}

Colesteatoma. Cirurgia. Diagnóstico por imagem. Classificação. Veterinária.

\section{Keywords}

Cholesteatoma. Surgery. Diagnostic imaging.

Classification. Veterinary.

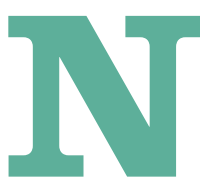

a rotina da clínica veterinária de cães é comum deparar-se com algumas doenças e afecções otológicas. Dentre essas, destaca-se o colesteatoma, uma manifestação secundária frequentemente associada a abordagens terapêuticas e tratamentos não eficazes da otite média crônica. Trata-se de uma doença relativamente rara, que acomete a orelha média do animal (LARSSON; LUCAS, 2016).

Os processos inflamatórios que acometem a orelha média, como a otite média crônica, costumam estar acompanhados de acúmulo de secreções e, no caso do colesteatoma, de uma formação cística epidermoide. É uma afecção costumeiramente não identificada nos atendimentos otológicos atuais, o que traz prejuízos significativos ao bem-estar animal, tendo como tratamento final a ablação total do conduto auditivo. A presença deste cisto também terá como resultados otalgia e incômodo local, além de poder afetar o nervo facial, levando à paralisia; pode, ainda, atingir a orelha interna, causando distúrbios de equilíbrio (KONIG; LIEBICH, 2011).

Este trabalho busca reunir informações sobre o colesteatoma, contribuindo com pesquisadores, estudantes e profissionais da Medicina Veterinária para conduzir melhor sua abordagem nos atendimentos clínicos e no respectivo tratamento. Como resultado final, espera-se um maior esclarecimento sobre a afecção, facilitando assim o seu diagnóstico e exatidão na conduta terapêutica.

\footnotetext{
1 Discente em Medicina Veterinária na Universidade Anhembi Morumbi, São Paulo/SP, Brasil.

2 Discente em Medicina Veterinária na Universidade Anhembi Morumbi, São Paulo/SP, Brasil.

3 Especializada em Medicina Veterinária e Docente da Universidade Anhembi Morumbi, São Paulo/SP, Brasil
} 


\section{Revisão anatômica}

A orelha - ou órgão vestibulococlear, em uma denominação mais rigorosa - tem como funções a audição e o equilíbrio, e é dividida em orelha externa, orelha média e orelha interna (KONIG; LIEBICH, 2011; LARSSON; LUCAS, 2016). Podemos visualizar suas particularidades na Figura 1.

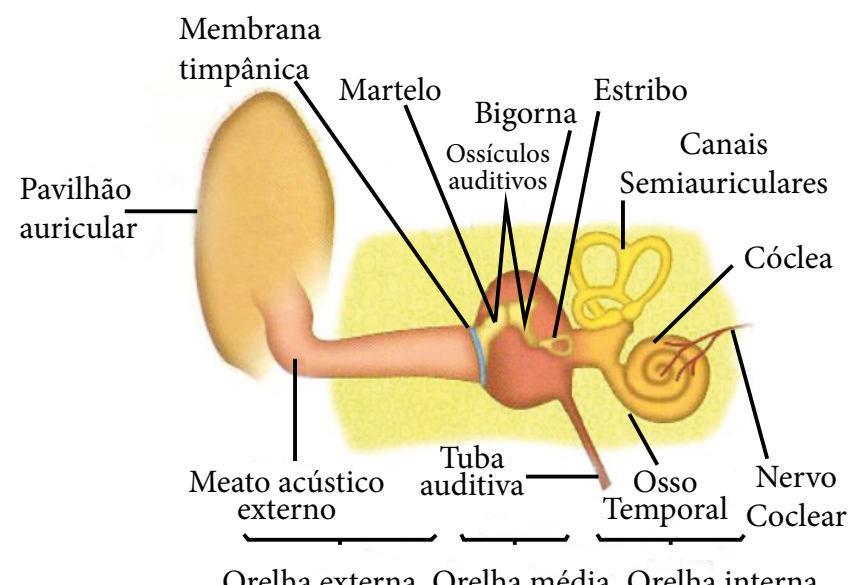

Figura 1 - Desenho representando o vestíbulo coclear de um cão. Fonte: Larsson; Lucas (2016).

A orelha externa possui uma porção vertical, o pavilhão auricular, uma estrutura móvel responsável por captar as ondas sonoras e transmiti-las à membrana timpânica; e outra porção horizontal, o meato acústico externo, que funciona como um canal até a membrana timpânica. A porção vertical e a horizontal da orelha externa são unidas em forma de L, formando um tubo cartilaginoso cônico (EVANS, 1993; KUMAR; ROMAN-AUERHALM, 2000; LARSSON; LUCAS, 2016).

A membrana timpânica é uma estrutura semitransparente, bem vascularizada, que separa a orelha externa da orelha média e está localizada na câmara dorsal da bula timpânica, região na qual são encontrados os três ossículos timpânicos (martelo, bigorna e estribo) e a janela da cóclea, estrutura que divide a orelha média da interna. A membrana timpânica pode ser visualizada com uso de um otoscópio (HEINE, 2004; KONIG; LIEBICH, 2011; LARSSON; LUCAS, 2016).

Já a orelha interna é responsável por receber os sinais auditivos, manter o equilíbrio e a posição dos olhos, tronco e membros. Está localizada no labirinto da porção petrosa do osso temporal e é composta por cóclea, vestíbulo e canais semicirculares (KUMAR; ROMAN-AUERHALM, 2000; HARVEY; HARARI; DELAUCHE, 2004).

As doenças graves ou inflamações acometendo a orelha média costumam estar acompanhadas por acúmulo de secreção. Também podem afetar o nervo facial, levando à paralisia, ou a orelha interna, causando distúrbios de equilíbrio (KONIG; LIEBICH, 2011).

\section{Definição}

O colesteatoma é uma doença rara, porém frequentemente associada com otite média crônica, aparecendo como uma manifestação secundária a abordagens terapêuticas e tratamentos não eficazes (FOSSUM, 2015).

Etimologicamente a palavra colesteatoma indica uma neoplasia contendo gordura ou cristais de colesterol. Porém, este termo se mostra impreciso, por tratar-se de um cisto epidermoide aural benigno, localizado geralmente na orelha média e com capacidade de migração e erosão das estruturas adjacentes, frequentemente encontrado nos ossos temporais. O cisto é formado por três componentes: conteúdo cístico, a debris de queratina; matriz, estroma de espessura variada; e perimatriz (TESTA et al., 2003; MAGALHÃES et al., 2005; BELOTTA et al., 2012).

$\mathrm{O}$ cisto epidermoide pode revelar-se por origem congênita ou adquirida, porém esta última é muito mais frequentemente relatada e mostra-se de duas formas possíveis, primária e secundária (FOSSUM, 2015).

\section{Etiopatogenia}

A etiopatogenia e epidemiologia do colesteatoma ainda não são bem definidas. (GRECI et al., 2011). A afecção pode ser congênita ou adquirida, sendo o último caso mais comum e classificado em primário e secundário (MUÑOZ RIVERA; MACIAS FERNÁNDEZ; CRUZ MEZA, 2007; FOSSUM, 2015).

Acredita-se que o colesteatoma congênito, embora raro, ocorra a partir de inclusão embrionária ou de remanescentes de células epiteliais. É localizado como uma massa branca perolada atrás de uma membrana timpânica intacta; na inexistência de fatores causais, como histórico de infeção aural, faz-se seu diagnóstico por exclusão (MUÑOZ RIVERA; MACIAS FERNÁNDEZ; CRUZ MEZA, 2007; FOSSUM, 2015).

Quanto à formação do colesteatoma adquirido, existem algumas teorias explicativas na literatura (MAGALHÃES et al., 2005):

- Teoria da metaplasia: devido a um processo inflamatório crônico, o epitélio cúbico se transforma em epitélio metaplásico queratinizado na orelha média (MAGALHÃES et al., 2005).

- Teoria da retração da membrana timpânica: a pressão negativa, por disfunção tubária ou infecção, causará deslocamento e perda de elasticidade da membrana timpânica, induzindo à formação de bolsas de retracção e promovendo a formação 
cística relatada como colesteatoma primário (MAGALHÃES et al., 2005; MUÑOZ RIVERA; MACIAS FERNÁNDEZ; CRUZ MEZA, 2007; ÁVILA et al., 2013).

- Teoria da migração do epitélio escamoso: é considerada como colesteatoma adquirido secundário. Ocorre a partir de uma perfuração na membrana timpânica, com a migração do epitélio escamoso para a cavidade timpânica (MAGALHÃES et al., 2005).

- Teoria da hiperplasia invasora: em resposta ao processo inflamatório local e atenuação em cascata das citocinas, ocorre uma proliferação de cones epiteliais nas camadas basais do epitélio queratinizado da membrana timpânica, levando à formação do colesteatoma (MAGALHÃES et al., 2005).

Nos cães, as duas teorias mais sugestiva para colesteatoma da orelha média são: a da migração e a da invaginação (GRECI et al., 2011). Na teoria da migração ocorre o perfuramento do tímpano, que permitirá a migração do epitélio escamoso estratificado do meato auditivo externo para a cavidade da orelha média infectada e, assim, produzirá condições necessárias para o desenvolvimento do colesteatoma. Já na teorida da invaginação, devido à pressão negativa e/ou à inflamação da orelha média, a pars flaccida e consequentemente a pars tensa da membrana timpânica retraem-se para a orelha média, em seguida a bolsa é lentamente preenchida pela queratina, formando assim o colesteatoma aural (MAGALHÃES et al., 2005; GRECI et al., 2011).

Além destas teorias, há alguns relatos de casos em que apareceu cisto epidermoide após procedimentos e cirurgias otológicas. Presume-se que o cisto tenha sido originado de elementos epidérmicos no momento da cirurgia, quando provavelmente foi implantado epitélio queratinizado na orelha (MAGALHÃES et al., 2005).

Em humanos também acredita-se que o colesteatoma pode ser congênito, resultante de um remanescente embrionário do tecido epitelial no conduto auditivo ou da persistência da formação epidermoide. O colesteatoma congênito também pode se formar como resultado da inclusão, migração, invasão do epitélio escamoso ou metaplasia do epitélio normal (MUÑOZ RIVERA; MACIAS FERNÁNDEZ; CRUZ MEZA, 2007; JANARDHAN et al., 2012).

\section{Predisposição}

Alguns trabalhos mostraram que, assim como em humanos, em que o sexo masculino é mais acometido, os cães machos também são mais predispostos a desenvolver a doença. A idade média do surgimento da doença é entre 4,5 a 10 anos (TRAVETTI et al., 2010; GRECI et al., 2011).
As raças mais predispostas seriam: pug, cocker spaniel, golden retriever e sem raça definida (SRD) (HARDIE; LINDER; PEASE, 2008; TRAVETTI et al., 2010).

Alguns relatos de caso cogitam a possibilidade de o colesteatoma ter se desenvolvido por conta da ablação total do conduto auditivo - osteotomia lateral da bula e osteotomia ventral da bula (HARDIE; LINDER; PEASE, 2008; SCHURENEMANN; OECHTERING, 2012).

\section{Sintomas}

Os sintomas mais comuns encontrados no animal com colesteatoma são: otalgia, dor ou inabilidade ao abrir a boca, head tilt, paralisia fácil, ataxia, dor à palpação da articulação temporomandibular e histórico de otite crônica (TESTA et al., 2003; HARDIE; LINDER; PEASE, 2008; GRECI et al., 2011; HARRAN et al., 2012). Também foi relatado colesteatoma aural em um bulldog francês, causando déficit sensorial do nervo trigêmeo (SCHURENEMANN; OECHTERING, 2012).

\section{Diagnóstico}

Podemos utilizar alguns meios de diagnóstico, como a tomografia computadorizada, vídeo-otoscopia, ressonância magnética (MAGALHÃES et al., 2005; FOSSUM, 2015). Porém, o exame histopatológico é o mais sugestivo para colesteatoma (NEWMAN et al., 2014).

O diagnóstico histológico do colesteatoma é feito pela identificação dos três componentes, restos de queratina, epitélio e tecido conjuntivo subepitelial (Figura 2). A presença de queratina disposta como massas ou flocos é altamente sugestiva de um colesteatoma subjacente, e o diagnóstico final baseia-se na tomografia computadorizada e nos achados cirúrgicos (NEWMAN et al., 2014).

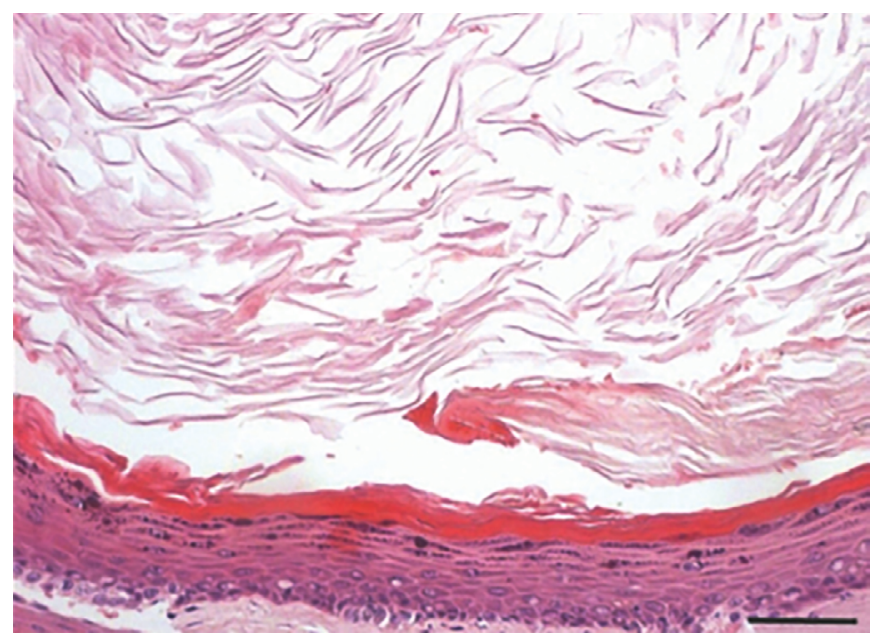

Figura 2 - Epitélio queratinizante intensamente hiperplástico, de camadas múltiplas (até 25 camadas de espessura) e com abundantes restos de queratina lamelar amorfa, sugestivo de colesteatoma.

Fonte: Greci et al. (2011) 
O exame de tomografia computadorizada é altamente sensível na averiguação de alterações do ouvido médio e é especialmente útil no diagnóstico do colesteatoma, devido às carcterísticas que apresenta (BELOTTA et al., 2012).

Embora este exame não possa ser utilizado sozinho como diagnóstico definitivo para colesteatoma aural, ele serve para distinguir um colesteatoma da orelha média, neoplasia e otite média, fornecendo informações relevantes ao médico-veterinário (TRAVETTI et al., 2010).

As neoplasias obscurecem o canal auditivo externo, podendo causar lise óssea da calota craniana adjacente e bula óssea. Podemos observar inchaço dos tecidos moles ao redor da orelha média e realce marcado do contraste (BELOTTA et al., 2012; DONALD, 2015).

$\mathrm{Na}$ otite média não encontramos rotineiramente o alargamento da cavidade timpânica (TRAVETTI et al., 2010). Já no caso de colesteatoma aural, veremos expansão da cavidade timpânica e esclerose (Figura 3) ou osteoproliferação do processo articular temporomandibular ipisilateral e paracondilar. É geralmente caracterizado por alterações ósseas graves no contorno da bula timpânica, como osteoproliferação, osteólise e osteosclerose. Pode causar também lise da parte petrosal do osso temporal, comcomplicações intracranianas e possível realce anelar (DONALD, 2015).

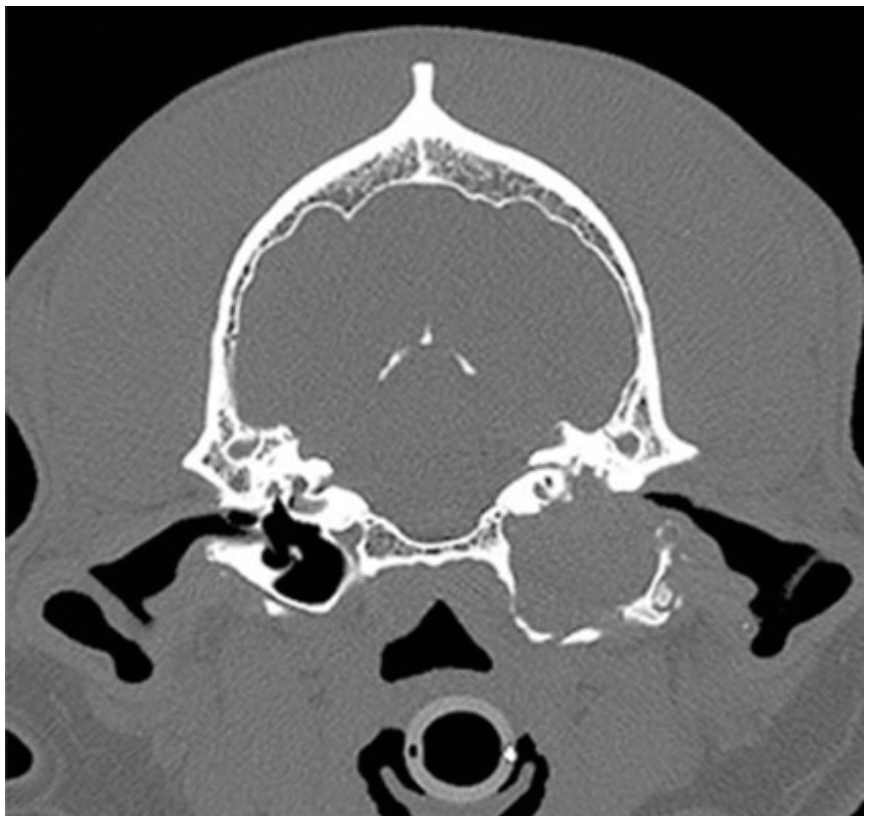

Figura 3 - Tomografia computadorizada de um cão com colesteatoma em conduto auditivo direito, em que se observam pressão da bula timpânica e necrose de expansão. Fonte: Donald (2015)

Durante a vídeo-otoscopia, a detecção de uma lesão de crescimento perolado ou de escalas brancas/amarelas na cavidade da orelha média deve alertar o clínico para a possível presença de colesteatoma da orelha média (FERLITO et al., 1997).
Na Medicina Veterinária, a presença de queratina sozinha ou de massas queratínicas na orelha média tem sido considerada adequada para estabelecer um diagnóstico de colesteatoma auricular (STURGES et al., 2006; HARDIE; LINDER; PEASE, 2008).

\section{Tratamento}

O único tratamento para o colesteatoma da orelha média é a cirurgia, mas a doença tende a recorrer. O objetivo da cirurgia é remover qualquer dentrito de queratina, epitélio escamoso estratificado e controlar a infecção (LITTLE et al., 1991; FERLITO et al., 1997; HARDIE; LINDER; PEASE, 2008).

Ablação Total do Canal Auditivo Osteotomia da Bula Lateral (TECALBO) é a técnica cirúrgica mais realizada (LITTLE et al., 1991; DAVIDSON; BRODIE; BREZNOCK, 1997; JACQUES; BOUCY, 1999; HARDIE; LINDER; PEASE, 2008).

Foi relatado que em três cães, durante a cirurgia de TECALBO, o material queratínico tinha aspecto esponjoso (Figura 4) (GRECI et al., 2011).

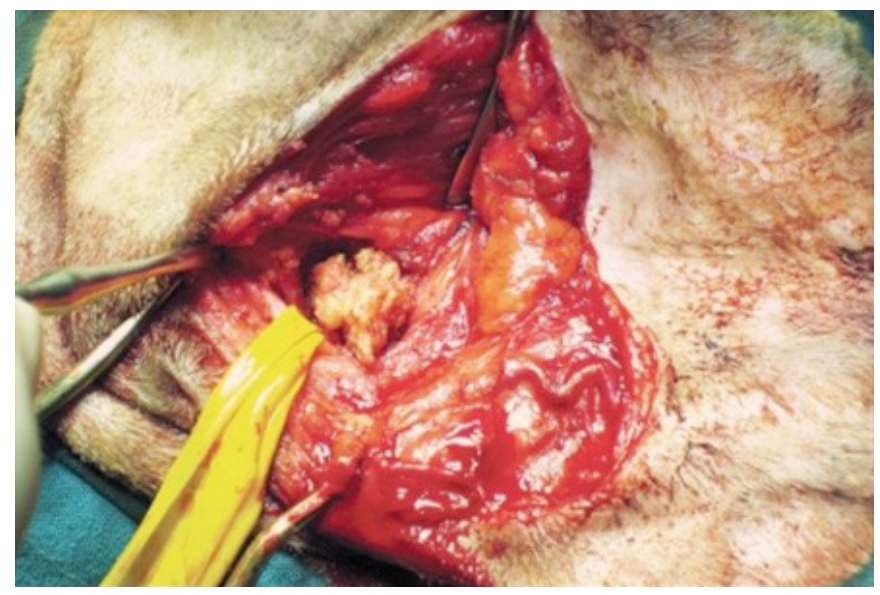

Figura 4 - Aparência grosseira do colesteatoma da orelha média durante a cirurgia. Fonte: Greci et al. (2011).

\section{Prognóstico pós-operatório}

Na medicina humana, pós-cirurgia de mastoidectomia radical modificada, os relatos de recidiva são baixos. Já na Medicina Veterinária, o risco de recorrência é alto para o colesteatoma, mesmo pós-cirurgia de TECALBO (FERLITO et al., 1997; ZANINI et al., 2005; HARDIE; LINDER; PEASE, 2008).

\section{Discussão}

Na busca por material para a construção deste trabalho, percebeu-se uma significante carência de informações a respeito da entidade patológica colesteatoma. Há poucos relatos de casos disponíveis na área da Veterinária. Porém, 
este trabalho, uma profunda revisão bibliográfica sobre a doença, concentra informações suficientes para que o médico-veterinário seja capaz de diagnosticar a doença durante o exame clínico e realize os consequentes exames complementares, ou ao menos construa uma suspeita diagnóstica de colesteatoma.

Acredita-se que o número de diagnósticos confirmados da doença em questão irá se revelar gradativamente, com o envolvimento do médico-veterinário com as informações disponíveis, a publicação de novos relatos de casos e a utilização adequada dos meios de diagnóstico da doença, como o exame histopatológico e a tomografia computadorizada, já citados.

\section{Considerações finais}

Com a avaliação de todo o material encontrado, entende-se a entidade patológica colesteatoma como uma formação cística epidermoide aural benigna, encontrada na orelha média do conduto auditivo, causada principalmente pela migração de epitélio escamoso e sua transformação em epitélio metaplásico queratinoso. No que se refere ao diagnóstico, em uma vista qualitativa, a avaliação por exame histopatológico de amostra coletada da orelha média e a tomografia computadorizada foram eleitas como ferramentas de excelência para constatar a presença do cisto em questão.

Como descrito neste trabalho, o tratamento mais indicado perante o diagnóstico de colesteatoma é a abordagem cirúrgica pela técnica TECALBO, caracterizada pela remoção da formação cística e de qualquer detrito de queratina. (*)

\section{Referências}

ÁVILA, A. F. A. et al. Avaliação por imagem dos colesteatomas da orelha média: ensaio iconográfico, Radiologia Brasileira, São Paulo, v. 46, n. 4, p. 247-251, 2013.

BELOTTA, A. F. et al. Colesteatoma: tomografia computadorizada e radiografia em cão com otite crônica. Veterinária e Zootecnia, Botucatu, v. 19, n. 3, p. 34-36, 2012.

DAVIDSON, E. B.; BRODIE, H. A.; BREZNOCK, E. M.; Removal of a cholesteatoma in a dog, using a caudal auricular approach. Journal of the American Veterinary Medical Association, Schaumburg, v. 211, n. 12, p. 1549-1553, 1997.

DONALD, E. T. Diagnóstico de radiologia veterinária. 6. ed. Rio de Janeiro: Elsevier, 2015. p. 125-129.

EVANS, H. E. The ear. In: MILLER, M. E.; EVANS, H. E. Miller's anatomy of the dog. 3. ed. Philadelphia: WB Saunders, 1993. p. 988-1008.
FERLITO, A. et al. Clinicopathological consultation: ear cholesteatoma versus choesterol granuloma. Annals of Otology, Rhinology, and Laryngology, St. Louis, v. 106, n. 1, p. 79-85, 1997.

FOSSUM, T. W. Cirurgia de ouvido. In: FOSSUM, T. W. Cirurgia de pequenos animais. 4. ed. Rio de Janeiro: Elsevier, 2015. p. 341-345.

GRECI, V. et al. Middle ear cholosteatoma in 11 dogs. The Canadian Veterinary Journal, Ottawa, v. 52, n. 6, p. 631-636, 2011.

HARDIE, E. M.; LINDER K. E.; PEASE, A. P. Aural cholesteatoma in twenty dogs. Veterinary Surgery, Malden, v. 37, n. 8, p. 763$770,2008$.

HARRAN, N. X. et al. Findings of a middle ear cholesteatoma in a dog. Journal of the American Animal Hospital Association, Lakewood, v. 48, n. 5, p. 339-343, 2012.

HARVEY, R. G.; HARARI, J.; DELAUCHE, A. Procedimentos para diagnóstico. In: HARVEY, R. G.; HARARI, J.; DELAUCHE, A. Doenças do ouvido em cães e gatos. Rio de Janeiro: Revinter, 2004. p. 70-72.

HEINE, A. P. Anatomy of the ear. The Veterinary Clinics of North America: small animal practice, Philadelphia, v. 34, n. 2, p. 379395, 2004.

JACQUES, D.; BOUCY, B. Un cas de holestéatome auriculaire chez un chien traité par ablation totale du conduit auditif associée à une ostéotomie latérale de la bulle tympanique. Pratique Médicale \& Chirurgicale de l'Animal de Compagnie, Paris, v. 34, p. 67-72, 1999.

JANARDHAN, N. et al. Congenital cholesteatoma of temporal bone with Bezold's abcess: case report, Indian Journal of Otolaryngology and Head \& Neck Surgery, Cham, v. 1, n. 1, p. 97-99, 2012.

KONIG, H. E.; LIEBICH, H.-G. Anatomia dos animais domésticos. 4. ed. Porto Alegre: Artmed, 2011.

KUMAR, A.; ROMAN-AUERHALM, M. R. Anatomy of the canine and feline ear. In: GOTTHELF, L. N. Small animal ear diseases. St. Louis: Saunders, 2000. p. 2-20.

LARSSON, C. E.; LUCAS, R. Tratado de medicina externa: dermatologia veterinária. São Caetano do Sul: Interbook, 2016.

LITTLE, C. J. et al. Inflammatory middle ear disease of the dog: the clinical and pathological features of cholesteatoma, a complication of otitis media. Veterinary Record, London, v. 128, n. 14, p. 319322, 1991.

MAGALHÃES, S. L. B. et al. Formação de cisto epidermóide (colesteatoma) com implantação de tecido epitelial junto ao osso femoral de ratos. Revista Brasileira de Otorrinolaringologia, São Paulo, v. 71, n. 2, p. 188-191, 2005. 
MUÑOZ RIVERA, C.; MACIAS FERNÁNDEZ, L. A.; CRUZ MEZA, F. C. Colesteatoma de oído. Anales de Radiología México, Ciudad de México, v. 6, n. 1, p. 71-77, 2007.

NEWMAN, A. W. et al. Cholesteatoma and meningoencephatilis in a dog with cronic otitis externa. Veterinary clinical pathology, New Jersey, v. 44, n. 1, p. 1-7, 2014.

SCHURENEMANN, R. M.; OECHTERING, G. Cholesteatoma after lateral bulla osteotomy in two brachycephalic dogs. Journal of the American Animal Hospital Association, Lakewood, v. 48, n. 4, p. 261-268, 2012.

STURGES, B. K. et al. Clinical signs, magnetic resonance imaging features, and outcome after surgical and medical treatment of otogenic intracranial infection in 11 cats and 4 dogs. Journal of Veterinary Internal Medicine, Philadelphia, v. 20, n. 3, p. 648656, 2006.

TESTA, J. R. G. et al. Colesteatoma causando paralisia facial. Revista Brasileira de Otorrinolaringologia, São Paulo, v. 69, n. 5, p. $657-662,2003$

TRAVETTI, O. et al. Computed tomography features of middle ear cholesteatoma in dogs. Veterinary Radiology \& Ultrasound, Oxford, v. 51, n. 4, p. 374-379, 2010.

ZANINI, F. D. et al. Colesteatoma de conduto auditivo externo: relato de caso. Revista Brasileira de Otorrinolaringologia, São Paulo, v. 71, n. 1, p. 91-93, 2005. 